\title{
microRNA function in left-right neuronal asymmetry: perspectives from $C$. elegans
}

\author{
Amel Alqadah ${ }^{1,2 \dagger}$, Yi-Wen Hsieh ${ }^{1 \dagger}$ and Chiou-Fen Chuang ${ }^{*}$ \\ ' Division of Developmental Biology, Cincinnati Children's Hospital Research Foundation, Cincinnati, OH, USA \\ ${ }^{2}$ Molecular and Developmental Biology Graduate Program, University of Cincinnati, Cincinnati, OH, USA
}

\section{Edited by:}

Laure Bally-Cuif, Centre National de la Recherche Scientifique, France

\section{Reviewed by:}

Hermona Soreq, The Hebrew University of Jerusalem, Israel Josh Gamse, Vanderbilt University, USA

\section{${ }^{*}$ Correspondence:}

Chiou-Fen Chuang, Division of Developmental Biology, Cincinnati Children's Hospital Research

Foundation, 240 Albert Sabin Way, Cincinnati, OH 45229, USA

e-mail: chiou-fen.chuang@cchmc.org

${ }^{\dagger}$ Amel Alqadah and Yi-Wen Hsieh have contributed equally to this work.
Left-right asymmetry in anatomical structures and functions of the nervous system is present throughout the animal kingdom. For example, language centers are localized in the left side of the human brain, while spatial recognition functions are found in the right hemisphere in the majority of the population. Disruption of asymmetry in the nervous system is correlated with neurological disorders. Although anatomical and functional asymmetries are observed in mammalian nervous systems, it has been a challenge to identify the molecular basis of these asymmetries. C. elegans has emerged as a prime model organism to investigate molecular asymmetries in the nervous system, as it has been shown to display functional asymmetries clearly correlated to asymmetric distribution and regulation of biologically relevant molecules. Small non-coding RNAs have been recently implicated in various aspects of neural development. Here, we review cases in which microRNAs are crucial for establishing left-right asymmetries in the C. elegans nervous system. These studies may provide insight into how molecular and functional asymmetries are established in the human brain.

Keywords: microRNA, neuronal asymmetry, sensory neurons, calcium signaling, C. elegans

\section{INTRODUCTION}

microRNAs (miRNAs) are endogenous 20-24 nt small non-coding RNAs that regulate gene expression through binding to complementary sequences in target messenger RNAs (mRNAs), leading to translational repression and/or cleavage of target mRNAs (Ambros, 2004; He and Hannon, 2004; Bartel, 2009; Chekulaeva and Filipowicz, 2009; Ghildiyal and Zamore, 2009). While most miRNAs downregulate gene expression, there are examples of miRNA-mediated upregulation of target gene expression during cell cycle arrest, suggesting that miRNA function is complex and context dependent (Vasudevan et al., 2007; Orom et al., 2008). miRNAs have been implicated in many aspects of development and disease including cell cycle, cell differentiation, apoptosis, life span, developmental timing, stress responses, neural development and regeneration, cancers, and neurodegenerative disorders (Boehm and Slack, 2005; Bushati and Cohen, 2007; Chang et al., 2009; Ambros, 2011; Sayed and Abdellatif, 2011; Zhang et al., 2011; Boulias and Horvitz, 2012; Cochella and Hobert, 2012a; Saito and Saito, 2012; Zou et al., 2012, 2013).

\section{FUNCTIONS OF miRNAs IN NEURAL DEVELOPMENT}

The importance of miRNAs in various aspects of neuronal development has been demonstrated in several animal models. In zebrafish, maternal-zygotic dicer mutants, that disrupt the processing of precursor miRNAs into mature miRNAs, display deleterious effects on the development of the brain, and injection of mature miR-430 rescues the early brain patterning defects (Giraldez et al., 2005). In mice, the neuron-specific miRNA miR-124 induces neuronal differentiation by directly targeting a global repressor of alternative pre-mRNA splicing and triggering a downstream switch to neuron-specific alternative splicing (Makeyev et al., 2007). In the Xenopus retina, a number of cell cycle related miRNAs target Xotx2 and Xvsx1 in early retinal progenitor cells to inhibit bipolar cell differentiation (Decembrini et al., 2009). In Drosophila, miR-9a targets senseless to inhibit neuronal fate in non-sensory organ precursors (Li et al., 2006). In C. elegans, the miRNA lin-4 targets the LIN-14 transcription factor to inhibit netrin-mediated axon attraction (Chang et al., 2004a; Zou et al., 2012), and the miRNA let-7 contributes to a developmental decline in neuronal regeneration (Zou et al., 2013). In addition, miRNAs $1 s y-6$, mir-273, and mir-71 function in asymmetric differentiation of two pairs of $C$. elegans sensory neurons, which will be discussed later (Johnston and Hobert, 2003; Chang et al., 2004b; Hsieh et al., 2012). Thus, miRNAs are important factors that control neuronal development across the animal kingdom.

\section{LEFT-RIGHT ASYMMETRY OF THE NERVOUS SYSTEM}

Although the nervous systems of animals are largely symmetric across the left-right axis, there have been several observations of anatomical and functional brain lateralization throughout the animal kingdom. For example, zebrafish display asymmetry in the epithalamus (Snelson and Gamse, 2009; Taylor et al., 2010); mice have been shown to have paw preferences, indicating the presence of a dominant hemisphere in motor control (Signore et al., 1991; Biddle et al., 1993); and the majority of humans have language centers such as Wernicke's and Broca's area located in the left hemisphere of the brain (Sun and Walsh, 2006). This lateralization of the nervous system is thought to be beneficial, as it allows for an increase of functional capacity (Rogers et al., 2012). 
Disruption of asymmetry in the brain is seen in a number of neurodevelopmental diseases, including dyslexia, schizophrenia, autism, Alzheimer's disease, and attention deficit/hyperactivity disorder (ADHD; Herbert et al., 2005; Derflinger et al., 2011; Oertel-Knochel and Linden, 2011; Renteria, 2012). In children diagnosed with ADHD, the prefrontal cortex was shown to have a loss in rightward asymmetric distribution of prefrontal cortex volume as compared to typically developing children (Shaw et al., 2009). In dyslexic patients, the planum temporale shows alteration in asymmetry, as the right temporale appears physically larger than the left counterpart (Galaburda et al., 1985).

There have been several reports on functional and anatomical nervous system asymmetries; however there have been comparatively fewer studies on identifying the molecular mechanisms that establish lateralization. Here we narrow our focus to the function and regulation of miRNAs in the development of neuronal asymmetry. The most interesting evidence of miRNAs in vertebrate neuronal asymmetry comes from the investigation of miRNA function in neocortex development. The simplest form of asymmetry is the division of a cell to give rise to two asymmetric fates. In the case of the mouse neocortex, a progenitor cell divides asymmetrically to give rise to a progenitor cell and a neuron. Asymmetric localization of the TRIM-NHL protein TRIM32 is observed in the daughter cell that becomes a neuron, while the cell lacking TRIM32 remains a progenitor (Schwamborn et al., 2009). TRIM32 increases the activity of specific miRNAs through binding of the RNAse argonaute-1 (Hammell et al., 2009; Schwamborn et al., 2009). It was further shown that the miRNA Let-7a, one of the TRIM32 targets, is required and sufficient for neuronal differentiation (Schwamborn et al., 2009).

A subset of zebrafish olfactory bulb output neurons called the mitral cells send axons asymmetrically to the right habenula, which is an asymmetric part of the brain where the higher olfactory processing center is located (Miyasaka et al., 2009; Taylor et al., 2010). Several molecules are asymmetrically expressed in adultborn olfactory neurons in the ventricular-subventricular zone (V-SVZ) of the zebrafish brain. The transcription factors Myt1 and Neurogenin 1 are predominantly expressed in the left V-SVZ, while DeltaA and hairy/enhancer of split-related protein (a Notch effector) are mainly expressed on the right side (Kishimoto et al., 2013). In addition, members of the miR-200 family are involved in the proper differentiation of olfactory neurons of both mice and zebrafish (Choi et al., 2008). It would be interesting to see whether the miR-200 family or other miRNAs influences the asymmetric and lateral projection of olfactory axons to the higher olfactory centers, and whether Myt1, Neurogenin1, and Delta/Notch are potential targets of miR-200.

A study found that 27 genes are differentially expressed in the embryonic human cerebral cortex, and the Lim domain transcription factor LMO4 is more abundant in the right perisylvian cortex than the left, and may be involved in asymmetric development of the cortex (Sun et al., 2005). The hypothesis that differential expression of these genes between left and right sides of the cortex may be regulated by miRNAs is plausible and worth further investigation.

Although there are limited reports of the involvement of miRNAs in the development of neuronal asymmetry in vertebrates, $C$. elegans has proved to be a powerful model organism to study lateralization of the nervous system due to its genetic amenability, as well as evidence of functional asymmetry having clear molecular correlates. In this review, we highlight two cases of the roles that miRNAs play in establishing left/right asymmetry in the C. elegans nervous system. Both involve the specification of two types of chemosensory neurons: the pair of amphid neurons, single cilliated endings (ASE) taste neurons, in which $l s y-6$ and mir-273 miRNAs are involved, and the pair of amphid wing "C" (AWC) olfactory neurons, where mir-71 is crucial for establishment of asymmetry.

\section{miRNAs IN TASTE NEURON ASYMMETRY}

Like other animals, the C. elegans nervous system appears generally symmetric. However, the pair of taste neurons, called ASE left (ASEL) and ASE right (ASER) displays molecular and functional asymmetries. The ASE neurons are located in the nerve ring of the nematode, which is the brain equivalent in the worm. Although the neurons are derived from different cell lineages, they are anatomically symmetric in terms of cell position, morphologies, and axonal projections (White et al., 1986). The ASEL neuron, however, differentially expresses the putative chemoreceptor $g c y-7$, while the ASER neuron expresses another putative chemoreceptor $g c y$-5 (Yu et al., 1997). The pair of ASE neurons also senses different chemicals, as the ASEL neuron functions to detect sodium, while ASER senses chloride (Pierce-Shimomura et al., 2001).

Over the past few years, there have been many studies investigating the molecular mechanism on how ASE asymmetry is established. Intriguingly, the first step of breaking symmetry of the taste neurons occurs several divisions before the ASEL and ASER neurons are born, during the early embryonic stage (Poole and Hobert, 2006). In the ASEL lineage, a pair of redundant T-box transcription factors TBX-37/38 are transiently expressed six cell divisions before the birth of ASEL (Good et al., 2004; Figure 1). These transcription factors work to "prime" a miRNA called $l s y-6$, which promotes the ASEL cell fate. This is achieved by binding of TBX-37/38 to a downstream primer element of $l s y-6$ and results in physical opening up of the $l s y-6$ chromatin (Cochella and Hobert, $2012 b$ ). The priming event is then "remembered" several cell divisions later in the ASEL mother cell. The open chromatin status of lsy-6 allows for the CHE-1 zinc finger transcription factor to bind to an upstream booster element of the $l s y-6$ locus (Cochella and Hobert, 2012b). This induces "boosting" of $l s y-6$ expression levels in the ASEL neuron. The "prime and boost" model is essential for establishing ASE asymmetry.

In the ASER lineage, a Delta/Notch signal in the ASER precursor cell causes the T-box transcription factors TBX-37/38 to be repressed (Good et al., 2004; Priess, 2005). Therefore, the lsy6 "priming" event does not occur in the ASER lineage, allowing the $l s y-6$ chromatin to remain in a compact form (Cochella and Hobert, 2012b). This in turn leads to the inability of the CHE-1 transcription factor to physically bind the upstream element of lsy-6, and no boosting of the miRNA expression levels occurs in ASER. Overall, this causes asymmetric distribution of the $l s y-6$ miRNA in the ASEL neuron.

The $l s y-6$ miRNA functions in a double negative feedback loop to control the asymmetry of ASEL/R neurons (Johnston et al., 


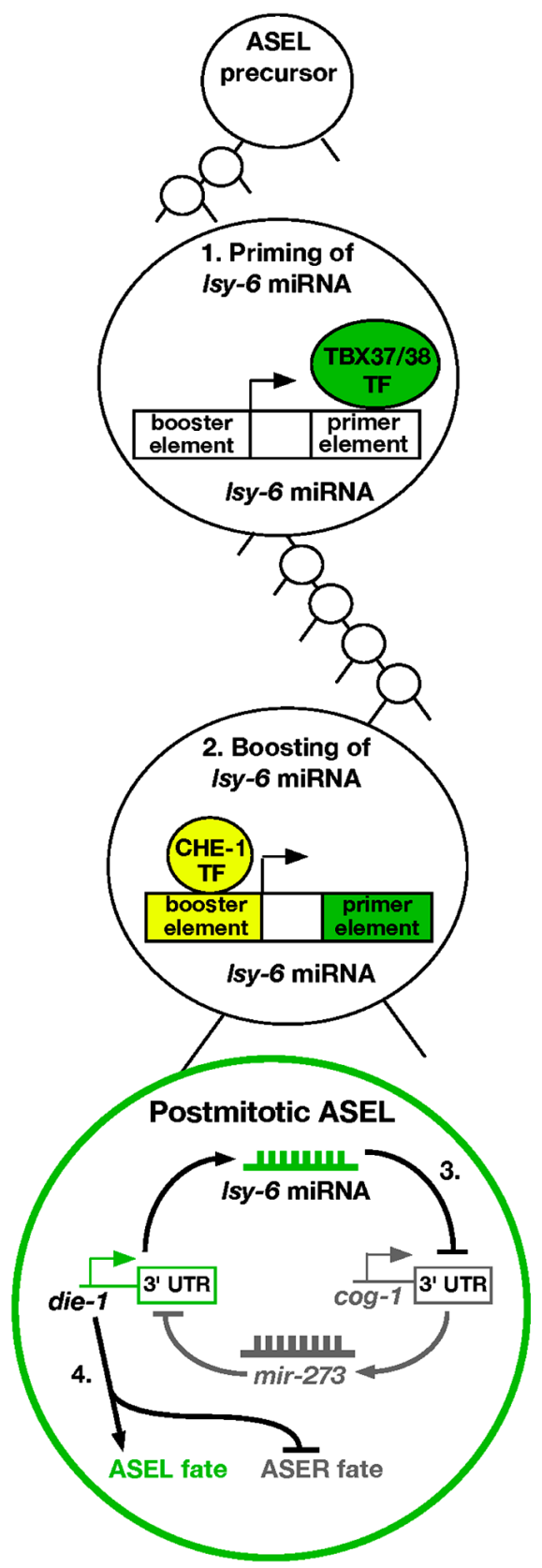

FIGURE 1 | Priming and boosting model of ASE asymmetry. ASEL: (1) The first step of promoting the ASEL cell fate occurs six cell divisions before the postmitotic ASEL is born. A transient pair of T-box transcription factors TBX-37/38 acts on the downstream primer element of Isy- 6 miRNA to open up the chromatin. (2) The priming event allows boosting of Isy- 6 miRNA levels by the $\mathrm{CHE}-1$ transcription factor in the ASEL mother cell, leading to high levels of the miRNA in the postmitotic ASEL neuron. (3) The Isy- 6 miRNA directly inhibits the ASER promoting transcription factor COG-1, and allows

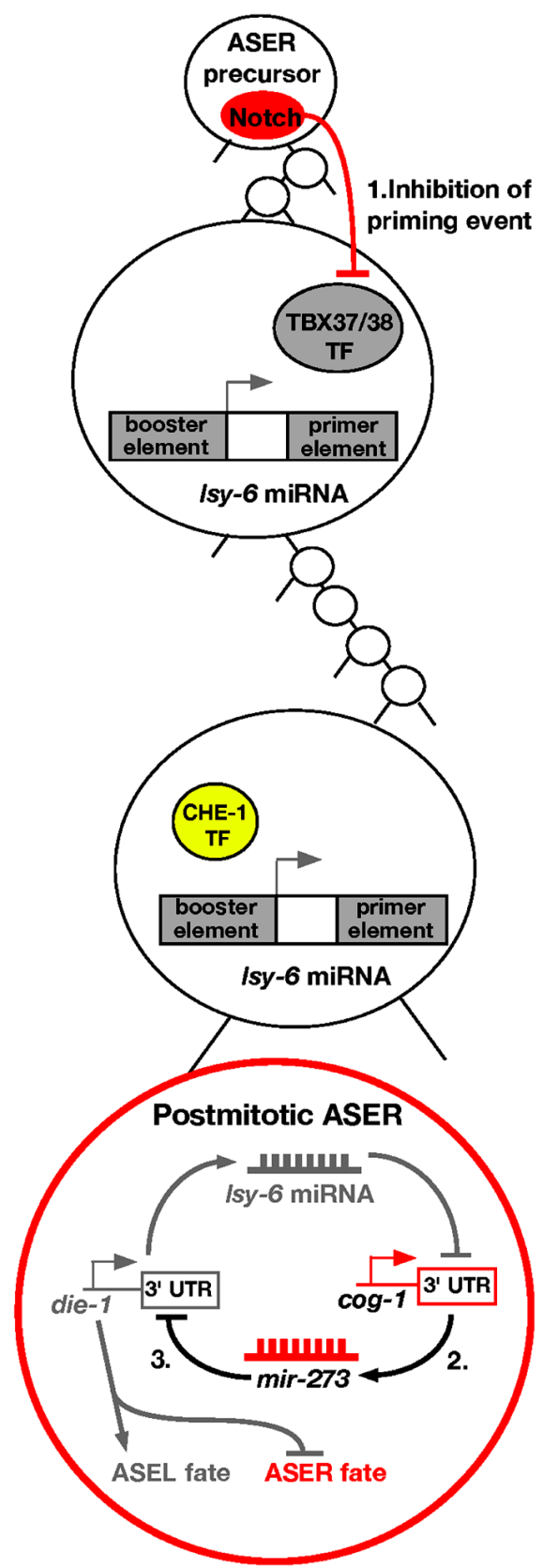

the ASEL promoting transcription factor DIE-1 to be expressed. (4) DIE-1 then activates ASEL effector genes and represses the ASER cell fate. ASER: (1) Notch is expressed in the ASER precursor and inhibits the Isy- 6 priming event. Therefore, the Isy- 6 miRNA expression level cannot be boosted as the chromatin remains compact. (2) Consequently, in the postmitotic ASER neuron, the COG-1 transcription factor is expressed, which activates another miRNA, mir-273. (3) mir-273 directly inhibits the ASEL promoting transcription factor DIE-1, and therefore promotes the ASER fate.
2005). In the ASEL neuron, lsy-6 directly represses an ASER promoting transcription factor COG-1, through physical binding of complementary bases in the $\operatorname{cog}-13^{\prime} \mathrm{UTR}$ (Johnston and Hobert, 2003). Repression of COG-1 allows for yet another transcription factor DIE-1 to be expressed, which is the output of the feedback loop. DIE-1 then activates ASEL effector genes and suppresses ASER effector genes (Johnston et al., 2005; Figure 1).

In the ASER neuron, lsy-6 expression is relatively low, and cannot effectively repress the COG-1 transcription factor. This allows activation of another miRNA, mir-273, which displays 
complementarity to the $3^{\prime} \mathrm{UTR}$ of the previously described die1 output (Chang et al., 2004b). mir-273 therefore represses DIE-1, resulting in the de-repression of the ASER fate (Figure 1). Mutating any of the factors involved results in a loss of asymmetry, with both cells adopting either the ASEL or ASER cell fate.

\section{miRNAs IN OLFACTORY NEURON ASYMMETRY}

Like the ASE neurons, the C. elegans AWC olfactory neurons are bilaterally symmetrical at the morphological level. However, AWC left (AWCL) and AWC right (AWCR) neurons express different odorant receptors and sense different odors (Figure 2; Troemel et al., 1999). The AWC ${ }^{\mathrm{ON}}$ neuron expresses the odorant receptor gene str-2 and specifically senses the odor butanone; while the contralateral $\mathrm{AWC}{ }^{\mathrm{OFF}}$ neuron expresses the odorant receptor gene $s r s x-3$ and specifically senses the odor pentanedione (Figure 2; Troemel et al., 1999; Wes and Bargmann, 2001; Bauer Huang et al., 2007). In wild-type animals, only one of the AWC neurons expresses str-2. AWC asymmetry is stochastic and coordinated, so that $50 \%$ of the worms in a population express str- 2 in AWCL, while the other 50\% express str-2 in AWCR (Troemel et al., 1999; Taylor et al., 2010). The default state of the AWC neurons is $\mathrm{AWC}{ }^{\mathrm{OFF}}$, which is specified by a calcium-regulated and microtubule-dependent MAP kinase pathway including UNC43/CaMKII, TIR-1/Sarm1, NSY-1/MAPKKK, and SEK-1/MAPKK (Figure 2; Sagasti et al., 2001; Tanaka-Hino et al., 2002; Chuang and Bargmann, 2005; Chang et al., 2011). NSY-4 claudin-like

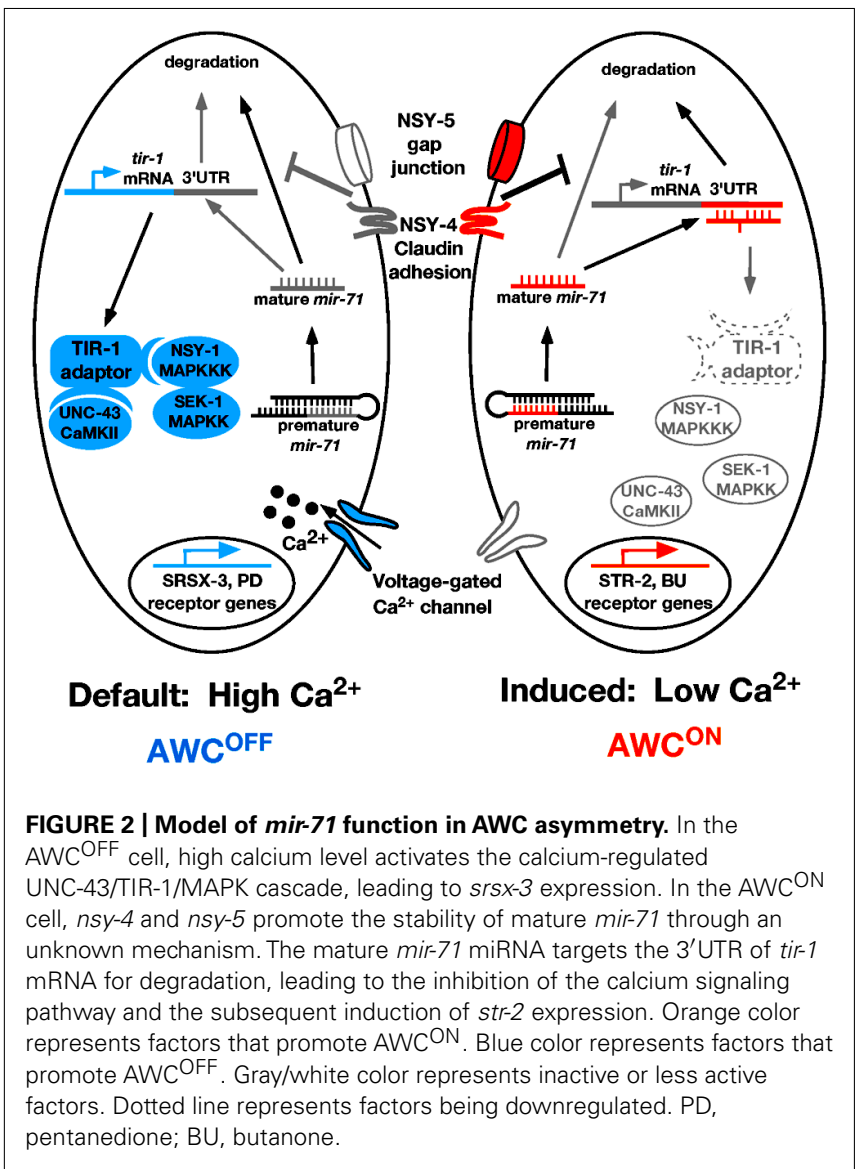

protein and NSY-5 gap junction protein act in parallel to inhibit the calcium signaling pathway in the induced $\mathrm{AWC}^{\mathrm{ON}}$ cell (Vanhoven et al., 2006; Chuang et al., 2007). In addition, intercellular calcium signaling between AWCs and non-AWC neurons via a NSY-5 gap junction-dependent neural network coordinates precise AWC asymmetry (Schumacher et al., 2012).

One of the important questions on AWC asymmetry is how the calcium signaling pathway is downregulated by $n s y-4$ and $n s y$ 5 in the AWC ${ }^{\mathrm{ON}}$ cell. A recent study showed that the miRNA mir-71 acts downstream of $n s y-4$ and $n s y-5$ to promote $A W C^{\mathrm{ON}}$ in a cell autonomous manner through inhibiting the expression of the calcium signaling adaptor protein gene tir-1 (Figure 2; Hsieh etal., 2012). The TIR-1/Sarm1 adaptor protein assembles a calcium-signaling complex to cell-autonomously specify the default AWC ${ }^{\text {OFF }}$ identity (Chuang and Bargmann, 2005). Thus downregulation of tir-1 expression by mir-71 is an efficient mechanism to inhibit calcium signaling in the cell becoming $\mathrm{AWC}^{\mathrm{ON}}$.

mir-71 is regulated at both transcriptional and posttranscriptional levels in AWC (Hsieh et al., 2012). At the transcriptional level, the expression level of mir-71 is higher in the $\mathrm{AWC}^{\mathrm{ON}}$ cell than in the AWC ${ }^{\mathrm{OFF}}$ cell. This transcriptional bias of mir-71 is not dependent on $n s y-4$ or $n s y-5$; thus, the mechanisms that regulate differential expression of mir-71 in the two AWC cells are yet to be elucidated. At the post-transcriptional level, the stability of mature mir-71 is dependent on $n s y-4$ and $n s y-5$. The C. elegans $5^{\prime} \rightarrow 3^{\prime}$ exoribonuclease XRN-2 has been shown to be involved in degradation of mature miRNAs (Chatterjee and Grosshans, 2009). It is possible that $n s y-4$ and $n s y-5$ may antagonize the $x r n$-2-mediated miRNA turnover pathway to increase the level of mature mir-71. However, RNA interference (RNAi) knockdown of $x r n-2$ did not cause a defect in AWC asymmetry, suggesting that the stability of mature mir-71 may be independent of $x r n-2$. In support of this idea, not all miRNAs accumulate in $x r n-2$ RNAi worms (Chatterjee and Grosshans, 2009), suggesting the existence of alternative miRNA turnover pathways that may be inhibited by $n s y-4$ and $n s y-5$.

\section{PERSPECTIVES}

Understanding the molecular mechanisms involved in establishing left-right asymmetry in the $C$. elegans nervous system can lay the groundwork for identifying the processes used in higher organisms, as the methods used may be evolutionarily conserved. Because of the highly conserved nature of miRNAs, insights into how they are involved to control asymmetric fates will help facilitate our understanding in vertebrate neuronal asymmetry. The involvement of miRNAs in asymmetry may also be reflective of the principles these small non-coding RNAs use in directing other neurodevelopmental processes.

\section{ACKNOWLEDGMENTS}

We thank Grethel Millington and Chieh Chang for comments on the manuscript. Amel Alqadah is supported by a Choose Ohio First Scholarship, Yi-Wen Hsieh by a NIH Organogenesis Training Grant, and Chiou-Fen Chuang by a Whitehall Foundation Research Award, an Alfred P. Sloan Research Fellowship, and a NIH R01GM098026 grant. 


\section{REFERENCES}

Ambros, V. (2004). The functions of animal microRNAs. Nature 431, 350-355. doi: 10.1038/ nature 02871

Ambros, V. (2011). MicroRNAs and developmental timing. Curr. Opin. Genet. Dev. 21, 511-517. doi: 10.1016/j.gde.2011.04.003

Bartel, D. P. (2009). MicroRNAs: target recognition and regulatory functions. Cell 136, 215-233. doi: 10.1016/j.cell.2009.01.002

Bauer Huang, S. L., Saheki, Y., VanHoven, M. K., Torayama, I., Ishihara, T., Katsura, I., et al. (2007). Left-right olfactory asymmetry results from antagonistic functions of voltage-activated calcium channels and the Raw repeat protein OLRN-1 in C. elegans. Neural Dev. 2, 24. doi: 10.1186/1749-81042-24

Biddle, F. G., Coffaro, C. M., Ziehr, J. E., and Eales, B. A. (1993). Genetic variation in paw preference (handedness) in the mouse. Genome 36, 935-943. doi: 10.1139/g93-123

Boehm, M., and Slack, F. (2005). A developmental timing microRNA and its target regulate life span in C. elegans. Science 310 , 1954-1957. doi: 10.1126/science. 1115596

Boulias, K., and Horvitz, H. R. (2012). The $C$. elegans microRNA mir-71 acts in neurons to promote germlinemediated longevity through regulation of DAF-16/FOXO. Cell Metab. 15, 439-450. doi: 10.1016/j.cmet. 2012.02.014

Bushati, N., and Cohen, S. M. (2007) microRNA functions. Annu. Rev. Cell Dev. Biol. 23, 175-205. doi: 10.1146/ annurev.cellbio.23.090506.123406

Chang, C., Hsieh, Y. W., Lesch, B. J., Bargmann, C. I., and Chuang, C. F. (2011). Microtubule-based localization of a synaptic calciumsignaling complex is required for left-right neuronal asymmetry in C. elegans. Development 138 , 3509-3518. doi: 10.1242/dev.06 9740

Chang, C., Yu, T. W., Bargmann, C. I., and Tessier-Lavigne, M. (2004a). Inhibition of netrin-mediated axon attraction by a receptor protein tyrosine phosphatase. Science 305, 103-106. doi: 10.1126/science.109 6983

Chang, S., Johnston, R. J. Jr., FrokjaerJensen, C., Lockery, S., and Hobert, O. (2004b). MicroRNAs act sequentially and asymmetrically to control chemosensory laterality in the nematode. Nature 430, 785-789. doi: 10.1038 /nature02752
Chang, S., Wen, S., Chen, D., and Jin, P. (2009). Small regulatory RNAs in neurodevelopmental disorders. Hum. Mol. Genet. 18, R18-R26. doi: 10.1093/hmg/ddp072

Chatterjee, S., and Grosshans, $\mathrm{H}$. (2009). Active turnover modulates mature microRNA activity in Caenorhabditis elegans. Nature 461, 546-549. doi: 10.1038/nature08349

Chekulaeva, M., and Filipowicz, W. (2009). Mechanisms of miRNAmediated post-transcriptional regulation in animal cells. Curr. Opin. Cell Biol. 21, 452-460. doi: 10.1016/j.ceb.2009.04.009

Choi, P. S., Zakhary, L., Choi, W. Y., Caron, S., Alvarez-Saavedra, E., Miska, E. A., et al. (2008). Members of the miRNA-200 family regulate olfactory neurogenesis. Neuron 57, 41-55. doi: 10.1016/j.neuron.2007.11.018

Chuang, C. F., and Bargmann, C. I. (2005). A toll-interleukin 1 repeat protein at the synapse specifies asymmetric odorant receptor expression via ASK1 MAPKKK signaling. Genes Dev. 19, 270-281. doi: 10.1101/gad.1276505

Chuang, C. F., Vanhoven, M. K., Fetter, R. D., Verselis, V. K., and Bargmann, C. I. (2007). An innexindependent cell network establishes left-right neuronal asymmetry in $C$. elegans. Cell 129, 787-799. doi: 10.1016/j.cell.2007.02.052

Cochella, L., and Hobert, O. (2012a). Diverse functions of microRNAs in nervous system development. Curr. Top. Dev. Biol. 99, 115-143. doi: 10. 1016/B978-0-12-387038-4.00005-7

Cochella, L., and Hobert, O. (2012b). Embryonic priming of a miRNA locus predetermines postmitotic neuronal left/right asymmetry in C. ele gans. Cell 151, 1229-1242. doi: 10.1016/j.cell.2012.10.049

Decembrini, S., Bressan, D., Vignali, R., Pitto, L., Mariotti, S., Rainaldi, G., et al. (2009). MicroRNAs couple cell fate and developmental timing in retina. Proc. Natl. Acad. Sci. U.S.A. 106, 21179-21184. doi: 10.1073/pnas.0909167106

Derflinger, S., Sorg, C., Gaser, C. Myers, N., Arsic, M., Kurz, A., et al. (2011). Grey-matter atrophy in Alzheimer's disease is asymmetric but not lateralized. J. Alzheimers Dis. 25, 347-357.

Galaburda, A. M., Sherman, G. F., Rosen, G. D., Aboitiz, F., and Geschwind, N. (1985). Developmental dyslexia: four consecutive patients with cortical anomalies. Ann. Neurol. 18, 222-233. doi: 10.1002/ ana. 410180210
Ghildiyal, M., and Zamore, P. D. (2009). Small silencing RNAs: an expanding universe. Nat. Rev. Genet. 10, 94-108. doi: 10.1038/nrg2504

Giraldez, A. J., Cinalli, R. M., Glasner, M. E., Enright, A. J., Thomson, J. M., Baskerville, S., et al. (2005). MicroRNAs regulate brain morphogenesis in zebrafish. Science 308, 833-838. doi: 10.1126/science. 1109020

Good, K., Ciosk, R., Nance, J., Neves, A., Hill, R. J., and Priess, J. R. (2004). The T-box transcription factors TBX-37 and TBX-38 link GLP-1/Notch signaling to mesoderm induction in $C$. elegans embryos. Development 131, 1967-1978. doi: 10.1242/dev.01088

Hammell, C. M., Lubin, I., Boag, P. R., Blackwell, T. K., and Ambros, V. (2009). nhl-2 modulates microRNA activity in Caenorhabdi tis elegans. Cell 136, 926-938. doi: 10.1016/j.cell.2009.01.053

He, L., and Hannon, G. J. (2004). MicroRNAs: small RNAs with a big role in gene regulation. Nat. Rev. Genet. 5, 522-531. doi: $10.1038 / \operatorname{nrg} 1379$

Herbert, M. R., Ziegler, D. A., Deutsch, C. K., O’Brien, L. M., Kennedy, D N., Filipek, P. A., et al. (2005). Brain asymmetries in autism and developmental language disorder: a nested whole-brain analysis. Brain 128, 213 226. doi: 10.1093/brain/awh330

Hsieh, Y. W., Chang, C., and Chuang, C. F. (2012). The microRNA mir71 inhibits calcium signaling by targeting the TIR-1/Sarml adaptor protein to control stochastic $\mathrm{L} / \mathrm{R}$ neuronal asymmetry in C. ele gans. PLoS Genet. 8:e1002864. doi: 10.1371/journal.pgen.1002864

Johnston, R. J. Jr., Chang, S., Etchberger, J. F., Ortiz, C. O., and Hobert, O. (2005). MicroRNAs acting in a double-negative feedback loop to control a neuronal cell fate decision. Proc. Natl. Acad. Sci. U.S.A. 102, 12449-12454. doi: 10.1073/pnas.0505530102

Johnston, R. J., and Hobert, O. (2003). A microRNA controlling left/right neuronal asymmetry in Caenorhabditis elegans. Nature 426, 845-849. doi: 10.1038 /nature02255

Kishimoto, N., Asakawa, K., Madelaine, R., Blader, P., Kawakami, K. and Sawamoto, K. (2013). Interhemispheric asymmetry of olfactory input-dependent neuronal specification in the adult brain. Nat. Neurosci. 16, 884-888. doi: 10.1038/ nn.3409

Li, Y., Wang, F., Lee, J. A., and Gao, F. B. (2006). MicroRNA-9a ensures the precise specification of sensory organ precursors in Drosophila.
Genes Dev. 20, 2793-2805. doi: 10.1101/gad.1466306

Makeyev, E. V., Zhang, J., Carrasco, M. A., and Maniatis, T. (2007). The MicroRNA miR-124 promotes neuronal differentiation by triggering brain-specific alternative pre-mRNA splicing. Mol. Cell 27, 435-448. doi: 10.1016/j.molcel.2007. 07.015

Miyasaka, N., Morimoto, K., Tsubokawa, T., Higashijima, S., Okamoto, H., and Yoshihara, Y. (2009). From the olfactory bulb to higher brain centers: genetic visualization of secondary olfactory pathways in zebrafish. J. Neurosci. 29, 4756-4767. doi: 10.1523/ JNEUROSCI.0118-09.2009

Oertel-Knochel, V., and Linden, D. E. (2011). Cerebral asymmetry in schizophrenia. Neuroscientist 17, 456-467. doi: 10.1177/ 1073858410386493

Orom, U. A., Nielsen, F. C., and Lund, A. H. (2008). MicroRNA$10 \mathrm{a}$ binds the $5^{\prime} \mathrm{UTR}$ of ribosomal protein mRNAs and enhances their translation. Mol. Cell 30, 460-471. doi: 10.1016/j.molcel.2008. 05.001

Pierce-Shimomura, J. T., Faumont, S., Gaston, M. R., Pearson, B. J., and Lockery, S. R. (2001). The homeobox gene lim-6 is required for distinct chemosensory representations in $C$. elegans. Nature 410, 694-698. doi: 10.1038/35070575

Poole, R. J., and Hobert, O. (2006). Early embryonic programming of neuronal left/right asymmetry in $C$. elegans. Curr. Biol. 16, 2279-2292. doi: 10.1016/j.cub.2006.09.041

Priess, J. R. (2005). "Notch signaling in the C. elegans embryo," in WormBook, ed. The C. elegans Research Community (Pasadena, CA: WormBook), 1-16. Available at: http://www.wormbook.org

Renteria, M. E. (2012). Cerebral asymmetry: a quantitative, multifactorial, and plastic brain phenotype. Twin Res. Hum. Genet. 15, 401-413. doi: 10.1017/thg.2012.13

Rogers, L. J., Vallortigara, G., and Andrew, R. J. (2012). Divided Brains: the Biology and Behaviour of Brain Asymmetries. Cambridge: Cambridge University Press.

Sagasti, A., Hisamoto, N., Hyodo, J., Tanaka-Hino, M., Matsumoto, K., and Bargmann, C. I. (2001). The CaMKII UNC-43 activates the MAPKKK NSY-1 to execute a lateral signaling decision required for asymmetric olfactory neuron fates. Cell 105, 221-232. doi: 10.1016/S00928674(01)00313-0 
Saito, Y., and Saito, H. (2012). MicroRNAs in cancers and neurodegenerative disorders. Front. Genet. 3:194. doi: 10.3389/fgene.2012.00194

Sayed, D., and Abdellatif, M. (2011). MicroRNAs in development and disease. Physiol. Rev. 91, 827-887. doi: 10.1152/physrev.00006.2010

Schumacher, J. A., Hsieh, Y. W., Chen, S., Pirri, J. K., Alkema, M. J., Li, W. H., et al. (2012). Intercellular calcium signaling in a gap junction-coupled cell network establishes asymmetric neuronal fates in C. elegans. Development 139, 4191-4201. doi: 10.1242/dev.083428

Schwamborn, J. C., Berezikov, E., and Knoblich, J. A. (2009). The TRIM-NHL protein TRIM32 activates microRNAs and prevents self-renewal in mouse neural progenitors. Cell 136, 913-925. doi: 10.1016/j.cell.2008.12.024

Shaw, P., Lalonde, F., Lepage, C., Rabin, C., Eckstrand, K., Sharp, W. et al. (2009). Development of cortical asymmetry in typically developing children and its disruption in attention-deficit/hyperactivity disorder. Arch. Gen. Psychiatry 66, 888-896. doi: 10.1001/archgenpsychiatry.2009.103

Signore, P., Nosten-Bertrand, M., Chaoui, M., Roubertoux, P. L., Marchaland, C., and Perez-Diaz, F. (1991). An assessment of handedness in mice. Physiol. Behav. 49, 701-704. doi: 10.1016/0031-9384(91)90305-8

Snelson, C. D., and Gamse, J. T. (2009). Building an asymmetric brain: development of the zebrafish epithalamus.
Semin. Cell Dev. Biol. 20, 491-497. doi: 10.1016/j.semcdb.2008.11.008

Sun, T., Patoine, C., Abu-Khalil, A., Visvader, J., Sum, E., Cherry, T. J., etal. (2005). Early asymmetry of gene transcription in embryonic human left and right cerebral cortex. Science 308, 1794-1798. doi: 10.1126/science. 1110324

Sun, T., and Walsh, C. A. (2006). Molecular approaches to brain asymmetry and handedness. Nat. Rev. Neurosci. 7, 655-662. doi: 10.1038/nrn1930

Tanaka-Hino, M., Sagasti, A., Hisamoto, N., Kawasaki, M., Nakano, S., Ninomiya-Tsuji, J., et al. (2002). SEK1 MAPKK mediates $\mathrm{Ca}^{2+}$ signaling to determine neuronal asymmetric development in Caenorhabditis elegans. EMBO Rep. 3, 56-62. doi: 10.1093/embo-reports/kvf001

Taylor, R. W., Hsieh, Y. W., Gamse, J. T., and Chuang, C. F. (2010). Making a difference together: reciprocal interactions in C. elegans and zebrafish asymmetric neural development. Development 137, 681-691. doi: 10.1242/dev.038695

Troemel, E. R., Sagasti, A., and Bargmann, C. I. (1999). Lateral signaling mediated by axon contact and calcium entry regulates asymmetric odorant receptor expression in C. elegans. Cell 99, 387398. doi: 10.1016/S0092-8674(00) 81525-1

Vanhoven, M. K., Bauer Huang, S. L., Albin, S. D., and Bargmann, C. I. (2006). The claudin superfamily protein nsy-4 biases lateral signaling to generate left-right asymmetry in C. elegans olfactory neurons. Neuron 51, 291-302. doi: 10.1016/j.neuron.2006.06.029

Vasudevan, S., Tong, Y., and Steitz, J. A. (2007). Switching from repression to activation: microRNAs can up-regulate translation. Science 318, 1931-1934. doi: 10.1126/science. 1149460

Wes, P. D., and Bargmann, C. I. (2001). C. elegans odour discrimination requires asymmetric diversity in olfactory neurons. Nature 410 , 698-701. doi: 10.1038/35070581

White, J. G., Southgate, E., Thomson, J. N., and Brenner, S. (1986). The structure of the nervous system of the nematode Caenorhabditis elegans. Philos. Trans. R. Soc. Lond. B Biol. Sci. 314, 1-340. doi: 10.1098/rstb.1986.0056

Yu, S., Avery, L., Baude, E., and Garbers, D. L. (1997). Guanylyl cyclase expression in specific sensory neurons: a new family of chemosensory receptors. Proc. Natl. Acad. Sci. U.S.A. 94, 3384-3387. doi: 10.1073/pnas.94.7.3384

Zhang, X., Zabinsky, R., Teng, Y., Cui, M., and Han, M. (2011). microRNAs play critical roles in the survival and recovery of Caenorhabditis elegans from starvation-induced L1 diapause. Proc. Natl. Acad. Sci. U.S.A. 108, 17997-18002. doi: 10.1073/pnas. 1105982108

Zou, Y., Chiu, H., Domenger, D. Chuang, C. F., and Chang, C. (2012). The lin-4 microRNA targets the LIN14 transcription factor to inhibit netrin-mediated axon attraction. Sci.
Signal. 5, ra43. doi: 10.1126/scisignal.2002437

Zou, Y., Chiu, H., Zinovyeva, A., Ambros, V., Chuang, C. F., and Chang, C. (2013). Developmental decline in neuronal regeneration by the progressive change of two intrinsic timers. Science 340, 372-376. doi: 10.1126/science. 1231321

Conflict of Interest Statement: The authors declare that the research was conducted in the absence of any commercial or financial relationships that could be construed as a potential conflict of interest.

Received: 25 June 2013; accepted: 01 September 2013; published online: 23 September 2013.

Citation: Alqadah A, Hsieh Y-W and Chuang C-F (2013) microRNA function in left-right neuronal asymmetry: perspectives from C. elegans. Front. Cell. Neurosci. 7:158. doi: 10.3389/fncel.2013. 00158

This article was submitted to the journal Frontiers in Cellular Neuroscience.

Copyright (C) 2013 Alqadah, Hsieh and Chuang. This is an open-access article distributed under the terms of the Creative Commons Attribution License (CC BY). The use, distribution or reproduction in other forums is permitted, provided the original author(s) or licensor are credited and that the original publication in this journal is cited, in accordance with accepted academic practice. No use, distribution or reproduction is permitted which does not comply with these terms. 